\title{
Turbulent kinetic energy and temperature variance dissipation in laboratory generated Rayleigh-Bénard turbulence designed to study the distortion of light by underwater microstructure fluctuations
}

\author{
Silvia Matt, Weilin Hou, Wesley Goode \\ Oceanography Division \\ Naval Research Laboratory \\ Stennis Space Center, MS 39529, USA \\ silvia.matt@nrlssc.navy.mil
}

\author{
Damien Josset \\ NRC Research Associate \\ Naval Research Laboratory \\ Stennis Space Center, MS 39529, USA
}

\begin{abstract}
Small-scale variation in temperature and salinity can lead to localized changes in the index of refraction and can distort electro-optical (EO) signal transmission in ocean and atmosphere. This phenomenon is well-studied in the atmosphere and in this context is generally called "optical turbulence". Less is known about how turbulent fluctuations in the ocean distort EO signal transmissions, an effect that can impact various underwater applications, from diver visibility to active and passive remote sensing. To provide a test bed for the study of the impacts from turbulent flows on EO signal transmission, as well as to examine and mitigate turbulence effects, we set up a laboratory turbulence environment allowing the variation of turbulence intensity. Convective turbulence is generated in a large Rayleigh-Bénard type tank $(5 \mathrm{~m}$ by $0.5 \mathrm{~m}$ by $0.5 \mathrm{~m})$ and the turbulent flow is quantified using a suite of sensors that includes high-resolution Acoustic Doppler Velocimeter profilers (Vectrino Profiler) and fast thermistor probes (PME ConductivityTemperature probe). These measurements allow the characterization of turbulent kinetic energy and temperature variance dissipation rates in the tank, for different convective strengths. Optical image degradation in the tank is then assessed in relation to turbulence intensity. The turbulence measurements are further complemented by very high-resolution computational fluid dynamics simulations of convective turbulence emulating the tank environment. These numerical simulations supplement the sparse laboratory measurements, providing full fields of temperature and velocity in the tank. The numerical data compared well to the laboratory data and both conformed to the Kolmogorov spectrum of turbulence and the Batchelor spectrum of temperature fluctuations. The numerical model was able to qualitatively reproduce the turbulence fields observed in the laboratory tank. Quantitatively, the numerical simulations are consistent with the observed $\varepsilon$ in the tank, but do not fully resolve the temperature gradients and thus underestimate $\chi$. The unique approach of integrating optical techniques, turbulence measurements and numerical simulations can help advance our understanding of how to mitigate the effects of turbulence impacts on underwater optical signal transmission, as well as on the use of optical techniques to probe oceanic processes.
\end{abstract}

Keywords-optical turbulence; turbulence measurements; ADV; Rayleigh-Bénard tank; turbulent dissipation rate

\section{INTRODUCTION}

The term "optical turbulence" is widely used in atmospheric optics to describe the phenomenon of distortion of EO transmission due to variations in the index of refraction caused by changes of air temperature along the optical path. This is similar to the visual perturbations that can be observed over a hot road or burning candle. A similar phenomenon occurs underwater, and here, can be caused by variations in either temperature or salinity that are associated with turbulent microstructure. Most often, temperature fluctuations are the dominating factor affecting the index of refraction [1], except in the case of strong freshwater or salt water influence, such as in river outflows or estuaries, or possibly in surface lenses generated by rainfall. In this study, we neglect the influence of salinity and instead focus on the effect of temperature.

Underwater "optical turbulence" at sea was investigated in the 1970 s by [2], who used this term to describe "small inhomogeneities in the index of refraction of seawater, their origins, and the effects they have on underwater optical systems". The phenomenon has since received limited attention, despite its potential to affect a wide range of applications, from diver visibility to active and passive remote sensing of the ocean. More recently, [1] looked at the effect of "light scattering on oceanic turbulence" with numerical studies compared to measurements in a small laboratory tank. Two recent field studies aimed at characterizing naturally-occurring "optical turbulence" in the aquatic environment highlight the difficulties associated with collecting concurrent data on optics and turbulence in the ocean [3] or lakes [4].

The impact of turbulent fluctuations on optical signal transmission can be quantified using the so-called optical turbulence parameter, $\mathrm{S}_{\mathrm{n}}$, which is the oceanic equivalent of the atmospheric optical turbulence coefficient $C_{n}^{2}[5][6] . S_{n}$ is a function of turbulent kinetic energy and temperature variance dissipation rates, $\varepsilon$ and $\chi$, respectively:

$$
\mathrm{S}_{\mathrm{n}} \sim \chi \varepsilon^{-1 / 3}
$$


Thus, in order to estimate $S_{n}$ and thus the amount of image degradation from turbulence, we need information on both velocity and temperature fluctuations. These measurements need to be of sufficient resolution to allow inferring dissipation rates $\varepsilon$ and $\chi$.

To simplify the measurements and reduce the number of parameters involved, such as, f. ex., platform motion polluting the velocity data and scattering due to particles degrading the optical signal, which affect field data in particular, we developed a laboratory environment for the study of underwater "optical turbulence". The laboratory setup also allows us to vary turbulence intensity and thus generate a "controlled turbulence environment" in terms of parameter space. The laboratory experiments are supplemented by numerical simulations using computational fluid dynamics (CFD). The numerical simulations provide full fields of temperature and velocity and thus provide a better view of the large-scale flow field and distribution of turbulence parameters than could be gathered with the sparse laboratory measurements alone. In this paper, we present the setup and results from laboratory and numerical experiments in this turbulence environment designed for the study of underwater "optical turbulence".

\section{METHODS}

\section{A. Laboratory Setup}

The laboratory setup consists of a large acrylic tank which is $5 \mathrm{~m}$ long and has a cross section of $0.5 \mathrm{~m}$ by $0.5 \mathrm{~m}$ and is outfitted with stainless steel plates at the bottom and top that can be temperature controlled (Fig. 1, left). In this tank, convective Rayleigh-Bénard type turbulence is generated by heating and cooling the bottom and top, respectively. The strength of the convective turbulence in the tank is a function of the temperature difference across the tank and can be characterized in terms of the Rayleigh number, defined as

$$
\mathrm{Ra}=\mathrm{g} \alpha \Delta \mathrm{Td}^{3} /\left(v \mathrm{D}_{\mathrm{T}}\right)
$$

Where $\mathrm{g}$ is the acceleration due to gravity, $\alpha$ is the thermal expansion coefficient, $\Delta \mathrm{T}$ is the temperature difference between the plates, $\mathrm{d}$ is the distance between the plates, $v$ is the kinematic viscosity, and $\mathrm{D}_{\mathrm{T}}$ is the thermal diffusivity.
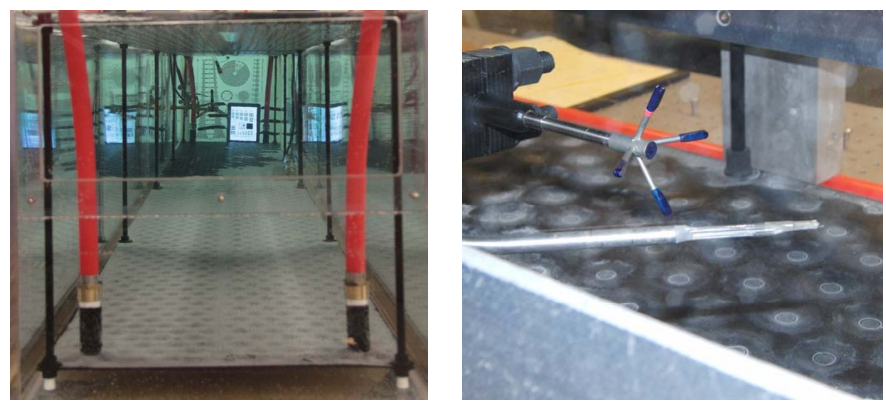

Fig. 1. Laboratory tank at NRLSSC. The photo on the right shows the Vectrino profiler ADV and the CT temperature probe.

This project was supported by ONR/NRL 73-4951/73-6604. Silvia Matt was supported by a NRC Research Associateship and a NRL Karle Fellowship.

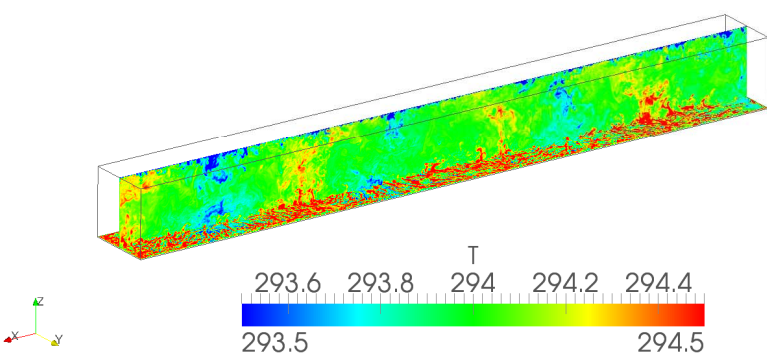

Fig. 2. Example of the "Numerical Tank" used to simulate Rayleigh-Bénard convection and emulating the laboratory tank setup. The temperature field (in $\mathrm{K})$ is shown and several convective plumes are visible.

The Rayleigh number of the flow can be changed by changing the plate temperatures, and thus the turbulence intensity can be varied. In our experiments, $\mathrm{Ra}$ ranges from $1.5 \cdot 10^{10}$ to around $4 \cdot 10^{10}$, corresponding to a temperature difference $\Delta \mathrm{T}$ between the plates of $6 \mathrm{~K}$ and $16 \mathrm{~K}$, respectively.

The turbulence in the tank is quantified by high-resolution Acoustic Doppler Velocimeter profilers (Nortek Vectrino Profiler) and fast thermistor probes (PME high-resolution conductivity-temperature (CT) probe) (Fig. 1, right). These instruments provide high-resolution velocity and temperature measurements, at 100 and $64 \mathrm{~Hz}$, respectively. Three ADVs and two CT probes were mounted in the tank and collected time series of high-resolution velocity and temperature/conductivity for the subsequent estimation of $\varepsilon$ and $\chi$. Data were collected at a sampling frequency of $100 \mathrm{~Hz}$ with the Vectrino Profiler and at $64 \mathrm{~Hz}$ with the CT probes, which were controlled by a Nortek Vector ADV in our setup. Turbulent kinetic energy dissipation rate $\varepsilon$ and temperature variance dissipation rate $\chi$ were calculated from the velocity and temperature measurements via spectral fitting to Kolmogorov spectra (for velocity) and Batchelor spectra (for temperature) and compared to values obtained from the numerical simulations of convective turbulence in the tank for comparable Rayleigh number and setup [7][8][9][10]. The turbulence data was put into the context of measurements of optical target clarity, by placing a high-speed imaging camera and active optical target, an iPad displaying optical resolution charts, at opposite ends of the tank, providing an optical path length of around $5 \mathrm{~m}$. To quantify the extent of image degradation from optical turbulence, an image quality metric, namely the Structural Similarity Index Method (SSIM) was applied [11]. The SSIM measures the similarity between two images, where one is considered to be of "perfect quality".

\section{B. Numerical Tank}

The sparse laboratory measurements (three Vectrino Profilers and two temperature probes) were complemented by CFD simulations of the convective tank. These threedimensional, very high-resolution, non-hydrostatic numerical simulations provide full fields of temperature and velocity for the estimation of turbulence parameters and their impact on the optics. 

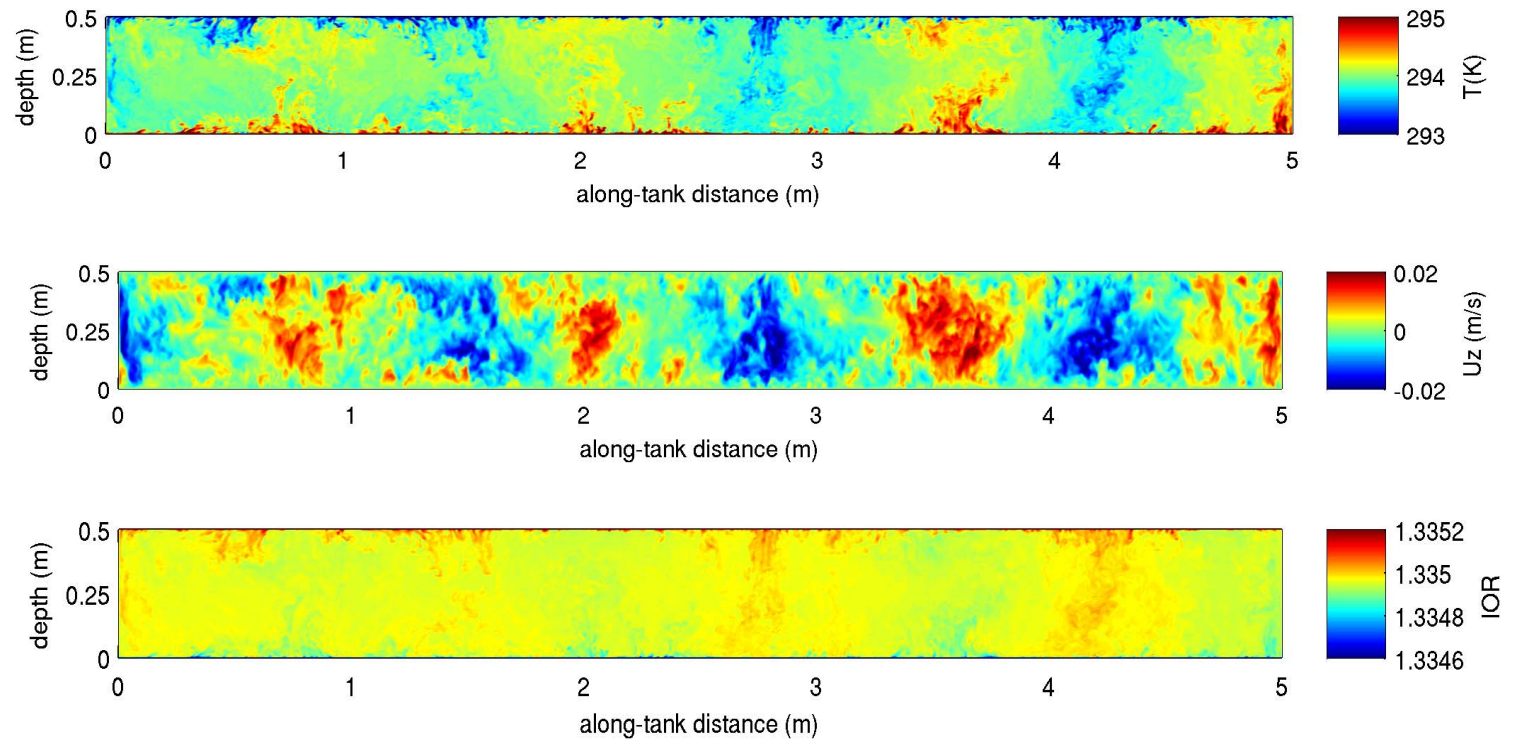

Fig. 3. Model results at time $t=1050$ s, showing a sideview of temperature $T$ (top), vertical velocity (Uz) (middle) and the Index of Refraction (IOR) calculated from the temperature field (bottom). The data is shown on the center plane of the tank.
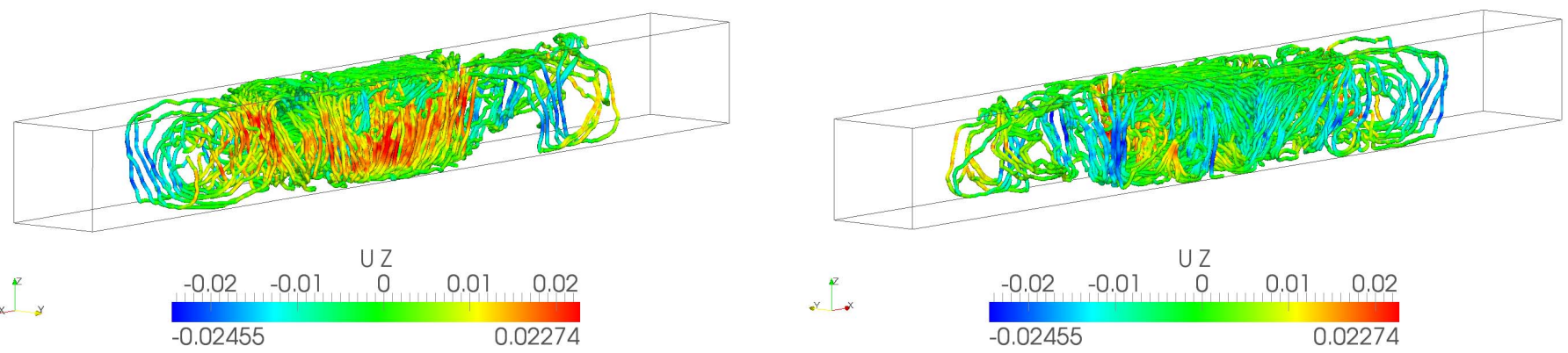

Fig. 4. Model results at time $\mathrm{t}=1050 \mathrm{~s}$, showing streamlines in the domain colored by vertical velocity $\mathrm{Uz}$ (top, in $\mathrm{m} / \mathrm{s}$ ). The bottom plot shows the same but looking at it from the other side of the tank ( $\mathrm{x}$ - and $\mathrm{y}$-axis reversed). The updrafts and downdrafts illustrate that there is a circulation in the tank in the cross-sectional direction. 

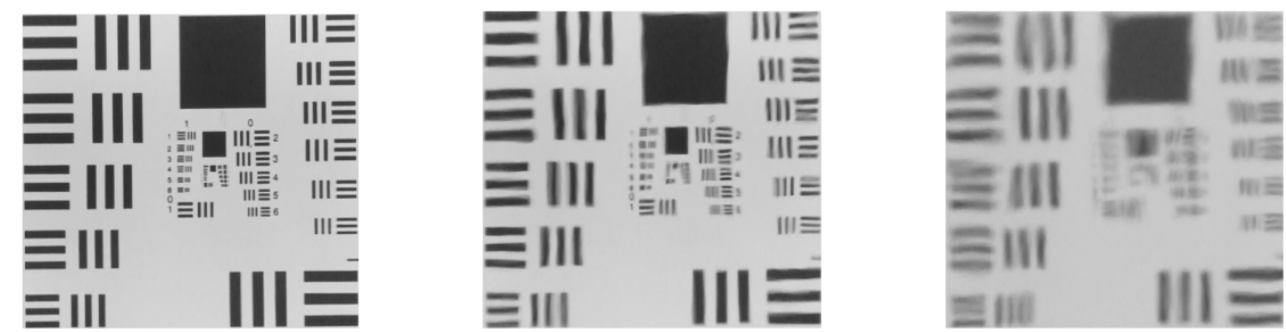

Fig. 5. Image degradation for different "optical turbulence" strengths. The images are taken with an optical path of $5 \mathrm{~m}$ (the length of the laboratory tank) of an optical resolution chart across a region of turbulence. Left is for a still tank (no turbulence), the middle is for a "strong" level of turbulence, and on the right is the highest level of turbulence ("extreme") we can achieve in the tank.

The numerical experiments were performed with the opensource CFD package OpenFOAM using a Large-Eddy Simulation (LES) approach. In LES, the larger-scale eddies in the flow are explicitly resolved, while the scales smaller than the grid-size are modeled [12]. The traditional Smagorinsky model was chosen as the sub-grid scale model [13]. Here, we present results from a very high-resolution, millimeter-scale simulation with $\Delta \mathrm{x}=\Delta \mathrm{y}=5 \mathrm{~mm}, \Delta \mathrm{z}=2.5 \mathrm{~mm}$, which corresponds to 20 million grid points in the $5 \mathrm{~m}$ by $0.5 \mathrm{~m}$ by $0.5 \mathrm{~m}$ domain (Fig. 2). Exploratory simulations at lower resolution $(\Delta x=\Delta y=\Delta z=1 \mathrm{~cm})$ were run on a modern, highperformance, dual six-core Linux desktop, whereas the production runs at the millimeter-scale resolution required High-Performance Computing resources at the DoD Supercomputing Resource Center, due to the high computational cost.

\section{RESULTS}

Temperature and velocity from the numerical simulations provide a view of the overall circulation in the tank, thus supplementing the sparse laboratory measurements. The simulations illustrate the convective cells that are established in the tank (Fig. 3). The size and number of the convective cells that develop in the tank are a function of the tank dimensions, in particular the tank height, since the water rises and sinks and gets diverted once it reaches the solid boundaries at the top and bottom. With a tank of depth $d=$ $0.5 \mathrm{~m}$ and length $\mathrm{L}=5 \mathrm{~m}$, we observe on the order of ten convective cells in our domain. In addition to the convective cells, secondary circulations, namely in the cross-sectional direction, develop in the domain (Fig. 4). These circulations can be visually confirmed in the laboratory when adding a tracer to the fully developed flow field, such as, for example, the seeding material needed to collect ADV data.

The model temperature fields and the index of refraction (IOR) calculated from these fields, using the empirical relation described in [14], provide an illustration of the disturbance expected to be encountered by an optical beam passing through the tank (Fig. 3).
Images from the laboratory show the image degradation due to optical turbulence for a case of "strong" (Fig. 5, center, $\Delta \mathrm{T} \approx 6 \mathrm{~K}$ ) and "extreme" optical turbulence (Fig. 5, right, $\Delta \mathrm{T}$ $\approx 16 \mathrm{~K})$. Here, particle scattering is secondary to the changes in the index of refraction due to temperature microstructure. Note that the effect of optical turbulence is more pronounced at the higher spatial frequencies. When applying the SSIM metric to a sequence of video images in order to quantify the extent of image degradation from optical turbulence, we confirm the differing amounts of image degradation for the two turbulence cases. The time series also hints at a difference in the frequency of variations between the two cases.

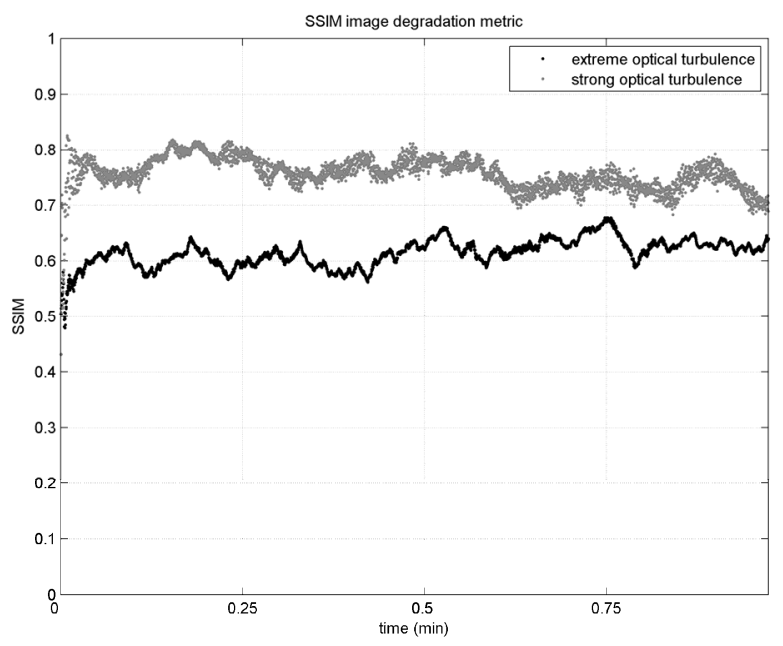

Fig. 6. Image degradation for different (optical) turbulence strength as shown in Fig. 5 quantified by applying the SSIM metric.

To quantify the turbulence in the tank and to estimate the turbulence parameters necessary to estimate the optical turbulence coefficient $S_{n}$, we calculate turbulent kinetic energy dissipation rates $\varepsilon$ and temperature variance dissipation rates $\chi$ from the data collected in the laboratory. These values are then compared to $\varepsilon$ and $\chi$ calculated from the numerical experiments. The numerical data compared well to the laboratory data and both conformed to the Kolmogorov 


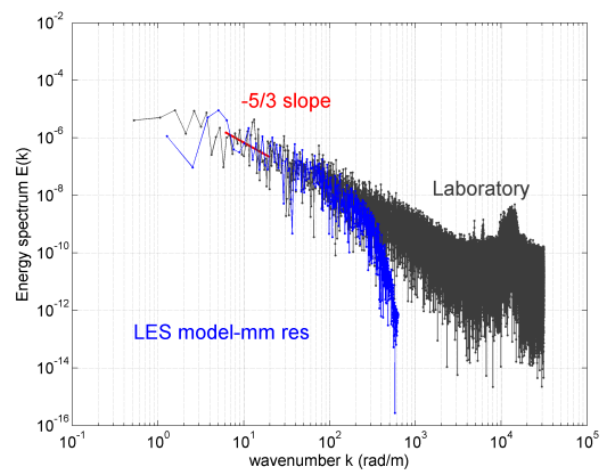

Fig. 7. Energy spectra (left) and temperature gradient spectra (right) from laboratory and model data. The energy spectra are shown only for the highest level of turbulence (compare to Fig. 5, right), since the spectra are not significantly changed for different convective turbulence strengths. The temperature gradient spectra do resolve the difference in turbulence strengths.

spectrum of turbulence and the Batchelor spectrum of temperature gradients (Fig. 7). The numerical model was able to qualitatively reproduce the turbulence fields observed in the laboratory tank. Quantitatively, the numerical simulations are consistent with the observed $\varepsilon$ in the tank, despite the fact that they do not resolve the spectrum down to the Kolmogorov microscale, which is on the order of $\mathrm{mm}$ for this flow.
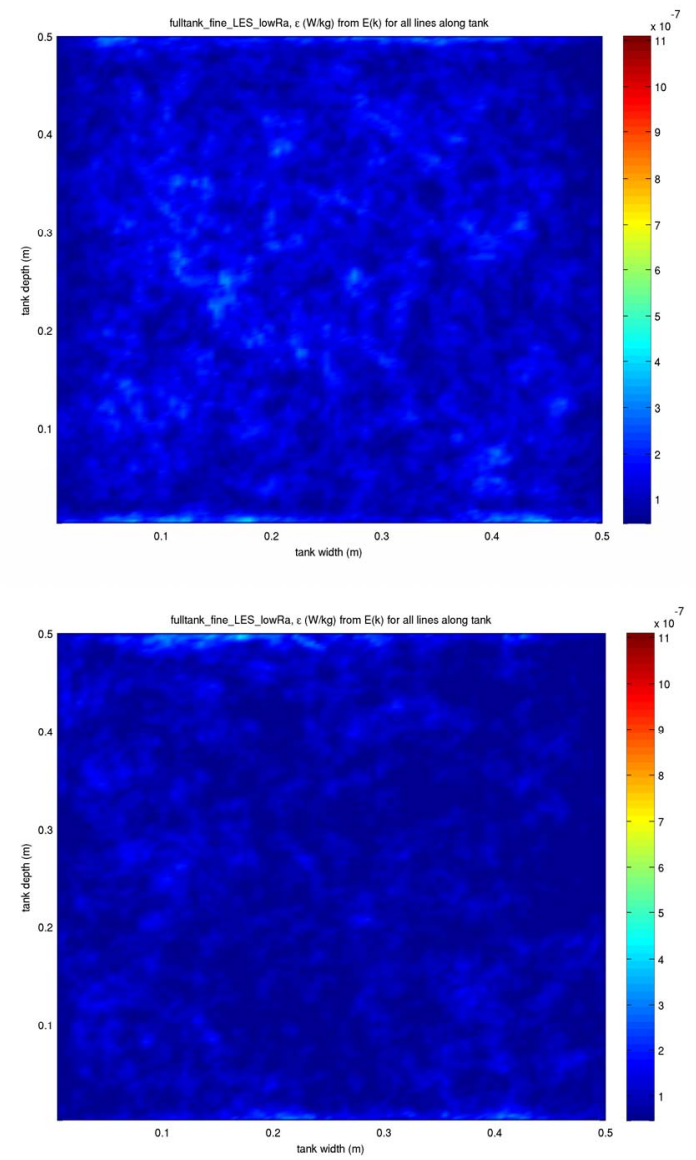

Fig. 8. Turbulent kinetic energy dissipation rate $\varepsilon$ from velocity section along the length of the tank at each $\mathrm{x}$-z-point, in W/kg. Left row: low Ra turbulence at times $\mathrm{t}=250 \mathrm{~s}$ (top) and $\mathrm{t}=1675 \mathrm{~s}$ (bottom). Right row: high Ra turbulence at times $\mathrm{t}=250 \mathrm{~s}$ (top) and $\mathrm{t}=1675 \mathrm{~s}$ (bottom).

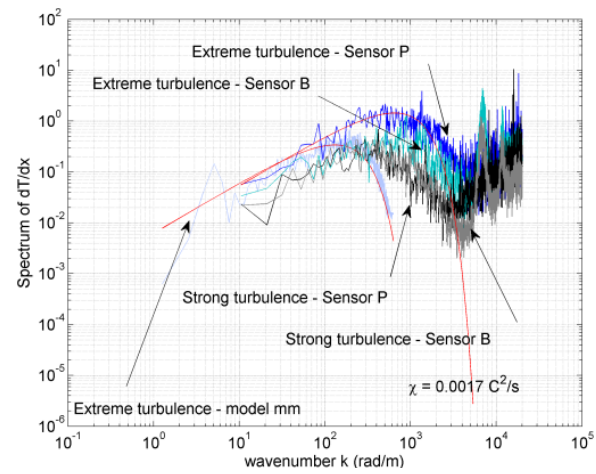

The laboratory data show that even for convective strength with dramatically different impact on the optics (Figs. 5, 6), the $\varepsilon$ in the tank remains within one order of magnitude. To illustrate how $\varepsilon$ is expected to vary across the tank crosssection, we can take the spectrum from the numerical model data at every $5 \mathrm{~m}$-long velocity section in the tank, for each $\mathrm{x}$ and z-position (Fig. 8).
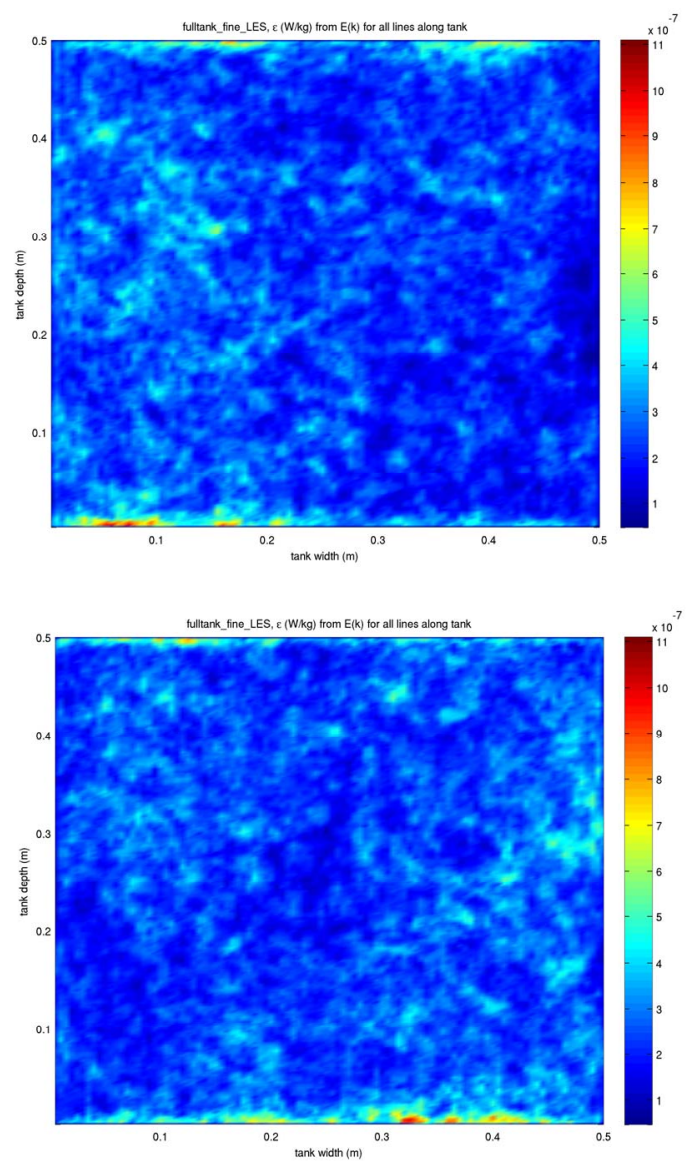
The value of $\varepsilon$ stays mostly within one order of magnitude across the tank. The variation near the boundaries due to boundary layer effects can be expected, and is consistent with the cross-sectional circulation seen in the velocity field. While the $\varepsilon$ from the model is consistent with the laboratory value, both are of $\mathrm{O}\left(10^{-7} \mathrm{~W} / \mathrm{kg}\right)$ for the experiments shown, the numerical simulations do not fully resolve the temperature gradients and thus underestimate $\chi$, even at the high resolution used in our experiments $(\Delta \mathrm{x}=\Delta \mathrm{y}=5 \mathrm{~mm}, \Delta \mathrm{z}=2.5 \mathrm{~mm} ; 20$ $\mathrm{M}$ grid points). The values of $\chi$ from both the laboratory and the numerical simulations show a significant difference for different convective turbulence strengths, but since the estimates from the numerical simulations are strongly resolution dependent and lower than the laboratory values for comparable turbulence strength, estimating the model sub-grid scale contribution to $\chi$ is of critical importance and is the subject of ongoing work. Since the effect on the optics is driven by changes in the index of refraction due to temperature variations, these results reemphasize the importance of characterizing in detail the temperature distribution to assess the impact of the turbulent fluctuations on the optics.

\section{CONCLUSIONS}

In order to study the impact of temperature microstructure on underwater optical signal transmission, we performed experiments in a controlled laboratory environment complemented by high-resolution, non-hydrostatic numerical simulations. The goal was to develop a setup where turbulence levels can be controlled and fully characterized. This setup, which allows for repeatable experiments under controlled conditions, can help us understand processes involved in optical turbulence and provide a platform for the testing of optical techniques to mitigate turbulence effects underwater. Optical turbulence is mainly due to temperature (or salinity) variations affecting the IOR of water, and to adequately describe the effect on the optics, it is particularly critical to resolve the temperature gradients. This can present a challenge in both the laboratory and the model, due to noise and resolution requirements, respectively. Further work is needed to address questions related to sub-grid scale contributions in LES to the rate of temperature variance dissipation. The unique approach of integrating optical techniques, turbulence measurements and numerical simulations can help advance our understanding of how to mitigate the effects of turbulence impacts on underwater optical signal transmission, as well as on the use of optical techniques to probe oceanic processes.

\section{ACKNOWLEDGMENT}

We thank Danielle Wain, Cynthia Bluteau and Barry Ruddick for sharing their data processing routines. Sarah Woods contributed to the tank design and early instrumental setup. We are grateful to Joe Calantoni for allowing us the use of his Vectrino II instrument. This project was supported by ONR/NRL 73-4951/73-6604. Silvia Matt was supported by a National Research Council (NRC) Research Associateship and a NRL Karle Fellowship.

\section{REFERENCES}

[1] D. Bogucki, J. Domaradzki, R. Ecke, and C. Truman, "Light scattering on oceanic turbulence," Appl. Opt. vol. 43, no. 30, pp. 5662-5668, 2004.

[2] G. D. Gilbert, and R. C. Honey, "Optical turbulence in the sea," Proc. SPIE 0024, Underwater Photo-Optical Instrumentation Applications III, 49, 1971.

[3] S. Matt, W. Hou, S. Woods, W. Goode, E. Jarosz, and A. Weidemann, "A Novel Platform to Study the Effect of Small-Scale Turbulent Density Fluctuations on Underwater Imaging in the Ocean," Methods in Oceanography, in press.

[4] W. Hou, S. Woods, E. Jarosz, W. Goode, and A. Weidemann, "Optical turbulence on underwater image degradation in natural environments," Appl. Opt, vol. 51, no. 15, pp. 2678-2686, 2012.

[5] W. Hou, "A simple underwater imaging model," Opt. Lett., vol. 34, no. 17, pp. 2688-90, 2009.

[6] M. C. Roggemann, and B. M. Welsh, Imaging through Turbulence, CRC Press, Boca Raton, Florida, USA, 1996.

[7] H. Tennekes and J. L. Lumley, A first course in turbulence. MIT Press, 1972.

[8] P. Rusello and E. Cowen, "Turbulent dissipation estimates from pulse coherent doppler instruments," in Current, Waves and Turbulence Measurements (CWTM), 2011 IEEE/OES 10th, pp. 167-172, 2011.

[9] B. Ruddick, A. Anis, and K. Thompson, "Maximum likelihood spectral fitting: The batchelor spectrum," J. Atmos. Oceanic Technol., vol. 17, pp. 1541-1555, 2000.

[10] J. Moum and J. Nash, "Mixing measurements on an equatorial ocean mooring," J. Atmos. Oceanic Technol., vol. 26, pp. 317-336, 2009.

[11] Z. Wang, A. C. Bovik, H. R. Sheikh, and E. P. Simoncelli, "Image quality assessment: From error visibility to structural similarity," IEEE Transactions on Image Processing, vol. 13, pp. 600-612, 2004.

[12] P. Sagaut, Large Eddy Simulation for Incompressible Flows, Springer, 1998.

[13] Smagorinsky, J., "General Circulation Experiments with the Primitive Equations. I. The Basic Experiment," Month. Wea. Rev., 91, 99-164, 1963.

[14] X. Quan and E. S. Fry, "Empirical equation for the index of refraction of seawater," Applied Optics, vol. 34, pp. 3477-3480, 1995. 